\title{
Predisposing factors of rhino-orbital-cerebral mucormycosis in patients with COVID 19 infection
}

\author{
Pradeep Pradhan $^{1}$ (D) Zaid Shaikh ${ }^{1} \cdot$ Abhijeet Mishra $^{1} \cdot$ C. Preetam ${ }^{1} \cdot$ \\ Pradipta Kumar Parida ${ }^{1}$ - Sourav Sarkar ${ }^{1}$ - Dillip Kumar Samal ${ }^{1}$. \\ Anindya Nayak $^{1} \cdot$ Srinivas Chadaram $^{1} \cdot$ Krishna Kinkar Das $^{1} \cdot$ Swagata Chakraborty $^{1}$. \\ Prity Sharma $^{1}$ - K. V. Aswathi ${ }^{1}$ - K. Vinusree ${ }^{1} \cdot$ Yash Mittal $^{1} \cdot$ Kalyana Sundaram $^{1}$. \\ Anurita Swarup $^{1} \cdot$ Asutosh Adhikari $^{1} \cdot$ Swathi Chenniappan $^{1} \cdot$ Anwer Shah $^{1}$
}

Received: 23 August 2021/Accepted: 15 September 2021/Published online: 25 September 2021

(C) Association of Otolaryngologists of India 2021

\begin{abstract}
The predisposing factors of invasive fungal disease in COVID 19 infection are still debatable because of the limited human understanding of the virus with the current literature. In this study, we have tried to correlate the various predisposing factors influencing the clinical profile and treatment outcomes in patients with covid associated mucormycosis (CAM). It is a retrospective analysis of cases of CAM during the second wave of COVID 19 infection, which was managed in the department of Otorhinolaryngology from Dec 1, 2020, to June 10, 2021. The detailed clinical, radiological and management of patients with CAM were collected, recorded, evaluated and correlated with the predisposing factors. Of the total, 46 patients, 44(95.65\%) were diabetic and 41 patients had a previous history of steroid intake. When clinical parameters were compared between blood sugar $<200 \mathrm{mg} / \mathrm{dl}$ and $>200 \mathrm{mg} / \mathrm{dl}$, the old and newly diagnosed diabetes mellitus in patients with CAM, there was no significant differences in any of the above clinical parameters $(p>0.05)$, except the hospital stay $(p=0,004)$. Steroid intake in patients with coexisting DM associated with CAM is considered the most important factor for the development of the CAM. There was are no significant difference in any of the clinical/treatment outcomes in patients with CAM with respect to the initial blood sugar, except for the hospital stay. A large sample size with a long-term follow-up period may be needed for a better
\end{abstract}

Pradeep Pradhan

padiapradhan@gmail.com

1 Department of ENT and Head Neck Surgery, All India Institute of Medical Sciences, Bhubaneswar, Odisha 751019, India understanding of common predisposing factors for the development of CAM.

Keywords COVID 19 - Mucormycosis .

Predisposing factors

\section{Introduction}

COVID-19 is a new disease entity caused by the novel coronavirus (SARS-CoV-2), first documented in China in December 2019 and subsequently driving a worldwide pandemic. The spectrum of symptoms of COVID 19 infection has been expanded from mild fever to severe pneumonia and often includes life-threatening secondary infections. This later could be due to the irrational use of systemic steroids for the management of COVID 19 infection. Again in a populated country like India, it is often challenging to provide hospital-based treatment to patients with mild to moderate covid pneumonia due to the limited infrastructure and unavailability of hospital beds where majorities of the patients are advised to self-treatment at home. Though steroid is considered as the magic drug for the management of the cytokine storm and the further sequelae in moderate to severe pneumonia, it acts as a double-edged sword by predisposing the patients to an immunocompromising state causing life-threatening infections, especially the COVID associated mucormycosis (CAM) in various subsites in head and neck. It typically contributes to the majority of the secondary fungal infection typically affecting the immunocompromised individual, including uncontrolled diabetes, iatrogenic immunosuppression, patients with organ transplantation and hematological malignancies [1]. As seen in the published literature, the incidence of CAM has been suddenly 
increased in the recent past after COVID 19 infections, significantly adding morbidity and mortality to the patients. Keeping in mind the rapid progression of the disease, early diagnosis of mucormycosis is a vital step in patients after the COVID 19 infection to prevent intraorbital and intracranial complications, which later accounts for about $50-80 \%$ of the total death [2]. Although different literature has been documented in the past showing the association of DM with the development of CAM, the detailed correlation of diabetes mellitus with respect to blood sugar is still not well defined in the past. In the present article, we have tried to correlate the clinical profile and the treatment outcomes with the common predisposing factors in patients with CAM.

\section{Materials and Methods}

All the patients of rhino-orbital/rhino-cerebral mucormycosis who were admitted and managed in the department of Otorhinolaryngology between Dec 1, 2020 to June 10 2021, were included in the study. The detailed clinical, radiological and treatment of patients with CAM were collected, recorded and evaluated from the medical records. In the clinical data, the symptoms, signs and associated comorbidities were documented. Radiological assessment was based upon the MRI/CT findings, showing the involvement of paranasal sinuses, orbit and the brain as demonstrated in Figs. 1, 2, 3. The history of diabetes mellitus, the preoperative blood sugar $(<200 \mathrm{mg} / \mathrm{dl}$ and $>200 \mathrm{mg} / \mathrm{dl}$ ), the duration of DM, and history of use of systemic steroids were noted. The association of the blood sugar level $(<200 \mathrm{mg} / \mathrm{dl}$ and $>200 \mathrm{mg} / \mathrm{dl}$, $)$ was correlated with the clinical profile, radiological involvement, and treatment outcomes of the patients affected with

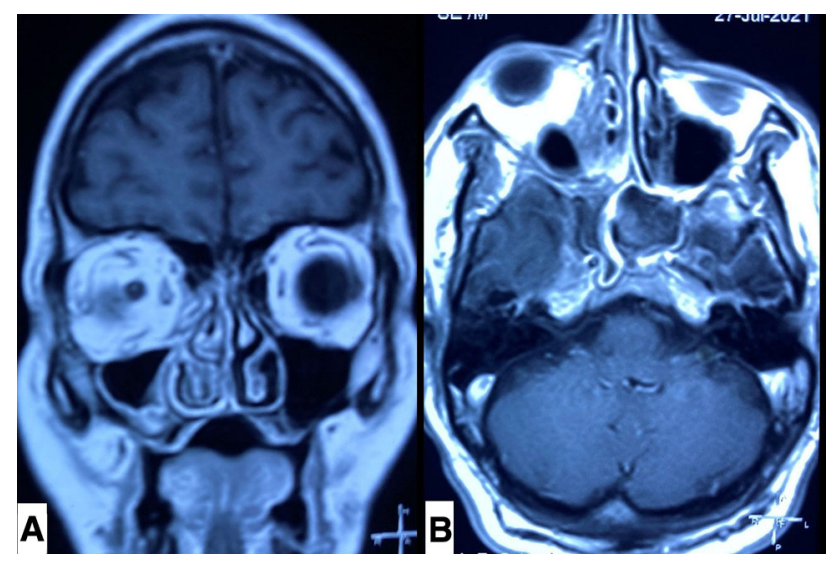

Fig. 1 Contrast-enhanced MRI, T1-weighted (A coronal cut, B axial cut) shows the contrast-enhanced shadow indicating the involvement of the intraconal compartment of the right orbit with involvement of maxillary and anterior ethmoid sinuses

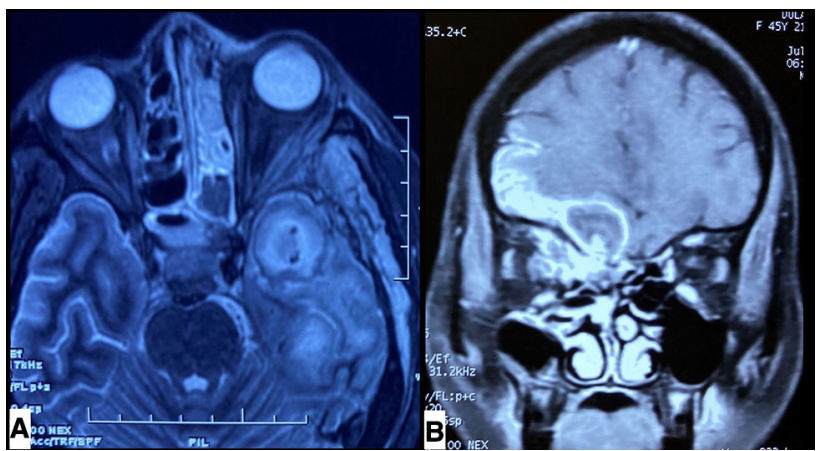

Fig. 2 Contrast enhanced MRI, T2 weighted (A, axial cut) shows hyperintense shadow involving left paranasal sinuses with a cerebral abscess in the left temporal lobe. B, T-1weighted(coronal cuts) shows right sinusitis with right frontal abscess, extending to the parietal brain

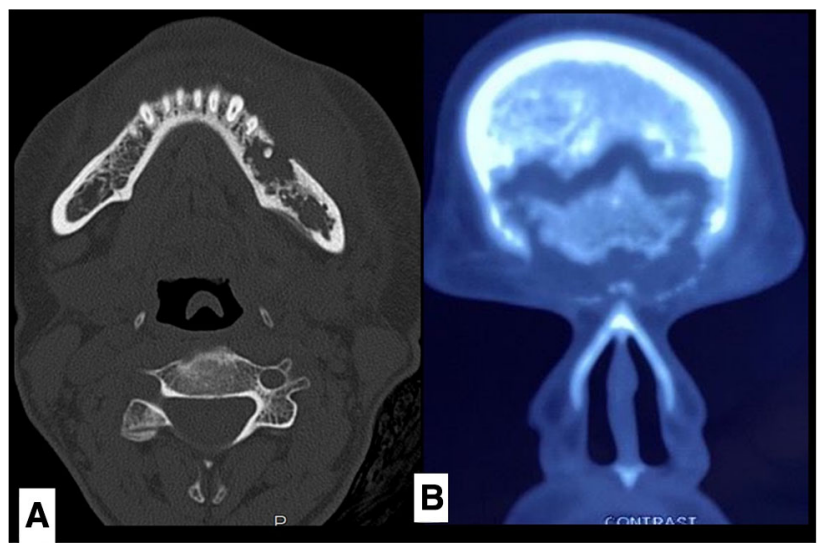

Fig. 3 Non-contrast MRI scan(A, axial cut) shows erosion of anterior arch of the mandible, B. (coronal cut) shows complete erosion of anterior table with sequestrum in the frontal sinus

CAM. Again, the clinical profile of the patients of CAM was compared with newly diagnosed versus previously diagnosed cases DM. The observed data were compared with the published data in the literature in the past.

\section{Results}

It was a retrospective analysis of 46 cases of CAM, which were managed in the department of Otorhinolaryngology from 1st December 2019 to May 10 2021. Of the total 46 patients, $40(86.95 \%)$ were male and 06(13.04\%) patients were females. The mean age of the patients was $48.80 \pm 10.83$ years (range $29-76$ years). Of the total 46 patients, $33(71.7 \%)$ were affected with rhino-orbital mucormycosis and 10 were rhino-cerebral mucormycosis. Two patients were found to affect the paranasal sinuses and one had isolated involvement of the mandible. The demographic profile and the clinical picture of the patients have been shown in Table 1. All the patients had a positive 
Table 1 Description of the demographic data in the study population [n $=46]$

\begin{tabular}{lll}
\hline Characteristics & Value/No of patients, N(\%) & Range \\
\hline Male & $40(86.95 \%)$ \\
Female & $06(13.04 \%)$ & $48.80 \pm 10.83$ \\
Mean age of patients (Year) & $16.46 \pm 10.05$ \\
Mean duration of the disease (days) & $44(95.65 \%)$ \\
Uncontrolled diabetes mellitus & $37(84.8 \%)$ \\
History of diabetes mellitus in the pre COVID period & $46(100 \%)$ \\
Positive COVID history & $41(89.1 \%)$ \\
History of steroid use & $220 \mathrm{mg} / \mathrm{dl}$ \\
Average blood sugar during admission & \\
Types of mucormycosis & $33(71.7 \%)$ \\
Rhino-orbital & $10(21.7 \%)$ \\
Rhino-cerebral & $1(2.2 \%)$ \\
Mandibular & $2(4.3 \%)$ \\
Sinonasal & $28 \pm 6$ \\
Hospital stay & $167-300 \mathrm{mg} / \mathrm{dl}$ \\
\hline
\end{tabular}

history of COVID 19 infection before presenting to the outpatient department. Of the 46 patients, 44(95.65\%) had a history of diabetes mellitus during their initial presentation to the department and two patients did not have a history of diabetes in the past. Almost all of the patients had uncontrolled blood sugar on initial evaluation, although none of them had presented with diabetic ketoacidosis. The average blood sugar was found to be 220 (range 167-300) as obtained with the initial investigation. Of the 44 patients with a history of diabetes patients, $37(80.43 \%)$ had been diagnosed first time during their treatment for COVID pneumonia and 7 had been diagnosed long before the COVID pneumonia. Of 46 CAM, Systemic steroid was prescribed in $41(89.1 \%$ ) patients, whereas 5 patients did not have a history of steroid ingestion for COVID infection. The steroid was given either in the form of methylprednisolone $(0.5-1 \mathrm{mg} / \mathrm{kg} / \mathrm{day})$ or dexamethasone $0.1-0.2 \mathrm{mg} / \mathrm{ml}$ ) for 5-7 days for COVID pneumonia. Rhino-orbital mucormycosis was the commonest variety encountered in the present study $(71.7 \%)$ and isolated involvement of the mandible was the least site to be affected $(2.2 \%)$ as demonstrated in the present study. Of $10(21.73 \%)$ patients with intracranial extension, 5 patients presented with frontal lobe abscess, 3 patients had involvement of the temporal lobe, one had a lesion in the brain stem and one presented with extradural abscess. Similarly, facial pain and swelling is the common symptom complained by the patient during their initial presentation to the outpatient department (Fig. 4). The diagnosis was made on correlating the suspicious history, clinical, radiological examination and later it was confirmed by the $\mathrm{KOH}$ smear from endoscopic nasal biopsy in each patient.

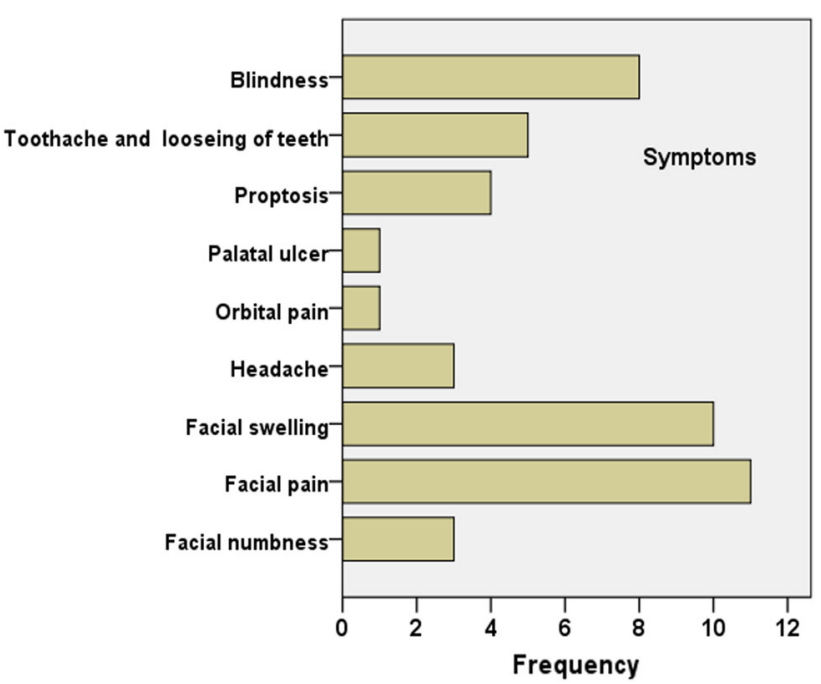

Fig. 4 Bar diagram which shows the clinical symptoms of patients with CAM when presented to the outpatient department

Surgical debridement followed by systemic amphotericin B was the standard treatment given to all the patients. Orbital exenteration as a part of surgical debridement was performed in 8 cases due to the complete loss of vision in the preoperative period. Majorities of the patients $(78.30 \%)$ underwent a combined surgical approach for complete clearance of the disease (Fig. 5). Surgery could not have been possible in 2 patients with CAM due to the critical medical condition who got supportive treatment in the intensive care unit. Again, two patients were referred after being operated on the outside, where patients were managed with injection amphotericin $\mathrm{B}$. When the primary presentation is correlated with respect to the blood sugar 


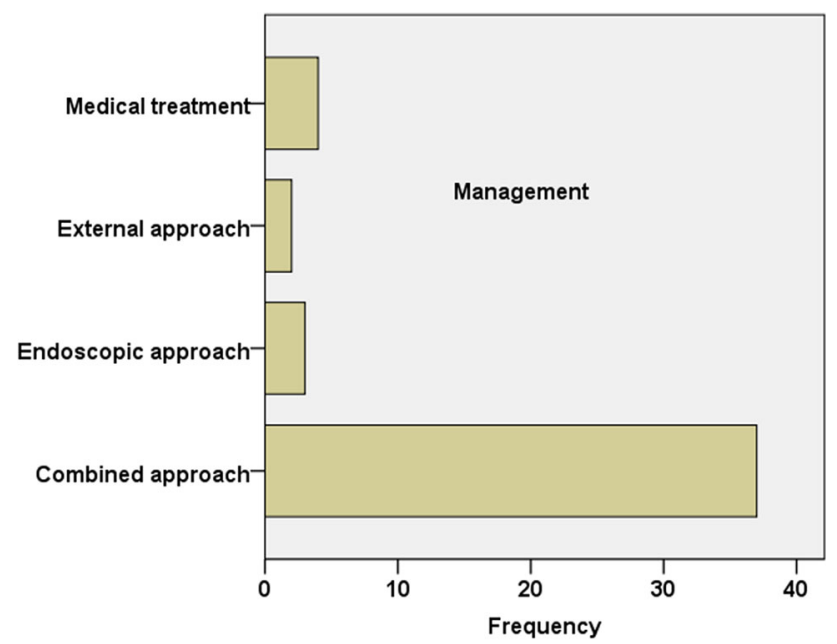

Fig. 5 Demonstrate the treatment protocol followed for patients with CAM

levels $(<200 \mathrm{mg} / \mathrm{dl}$ and $>200 \mathrm{mg} / \mathrm{dl})$ in patients with CAM, we did not find any statistical difference among them $(p=0.811)$. When the major sites of involvement, i.e., the orbit and the brain, were accounted and compared between patients the two sugar levels, the result was found to be insignificant $(p=0.849)$. Similar results have been obtained for the surgical approaches, orbital exenteration on comparing the sugar levels $(p=0.713$ and $p=0.520)$, respectively. There was no significant association between hyperglycemia to the affection of various subsites in the brain $(p>0.05)$. The average hospital stay was found to be 28 days (range 19-40 days). When the hospital stay was compared between two sugar levels, we found a significant difference between them $(p=0.004)$. Again the duration of the disease did not have a significant effect on the orbital/ cerebral Mucormycosis $(p>0.05)$. During the hospital stay, $31(73.80 \%)$ patients had undergone revision surgery during their treatment in the hospital. When the number of patients who underwent revision surgery was compared between the blood sugar levels/onset of DM, no significant difference was noted ( $p=0.673$ and 0.697 , respectively). Four patients with rhino-orbital involvement and 5 patients with rhino-cerebral mucormycosis have died during their treatment in the ward and there was no significant difference in the death rates between the blood sugar levels $(p=0.550)$. Again, when we compare the clinical parameters and patient outcomes between the old and newly diagnosed DM associated with CAM, we did not find any significant differences between any of the above parameters (Table 2).

\section{Discussion}

Mucormycosis is an angioinvasive disease caused by fungi of the order Mucorales like Rhizopus, Mucor, Rhizomucor, Cunninghamella and Absidia affecting various subsites in the head-neck region. The incident rate of mucormycosis varies from 0.005 to 1.7 per million populations [3]. The prevalence of mucormycosis in India is approximately 0.14 cases per 1000 population, about 80 times more prevalent than the developed countries. It is more often seen in immunocompromised individuals like diabetes mellitus, organ transplants, or patients receiving chemotherapy for malignant tumours. Amongst them, diabetes mellitus is considered a significant independent risk factor for rhinoorbital-cerebral mucormycosis [3]. Although it is a wellknown disease, the incidence of invasive fungal infection has been significantly increased with the onset of the second wave of COVID 19 infection. On reviewing the past literature, a large number of articles have been published in the past claiming the association of diabetes mellitus with CAM [4-11] and rhino-cerebral mucormycosis [12-16] in the COVID 19 infection. In spite of the confirmed association of DM with CAM, none of the articles have published in the past have documented the clear correlation of blood sugar with the clinical profile and treatment outcomes in patients with CAM. The results obtained in the present study was also very much similar to the published literature, where $44(95.65 \%)$ patients with CAM had a positive history of DM during their initial evaluation to the department and 2 patients were found to be non-diabetic Among the 44 patients with underlying DM, 37(80.43\%) were newly diagnosed during their treatment for COVID pneumonia, and 7 had had a history of DM before the development of COVID 19 infection. When the clinical parameters, like symptoms, sites of involvement, surgical approaches, orbital exenteration and death of the patients were compared between the blood sugar $<200 \mathrm{mg} / \mathrm{dl}$ and $>200 \mathrm{mg} / \mathrm{dl}$, we did not get a significant difference for any of the clinical parameters $(p>0.05)$. The only significant difference we obtained was the hospital stay i,e. patients with blood sugar levels $>200 \mathrm{mg} / \mathrm{dl}$ had significantly lesser hospital stay as compared to the patients with blood sugar $<200 \mathrm{mg} / \mathrm{dl}(p=0.004)$. This could be due to the better tolerability of medical treatment by the patients of CAM as a result of adequate preservation of the renal function. When the clinical parameters, like symptoms, sites of involvement, surgical approaches, orbital exenteration and death of the patients were compared between the newly diagnosed and previously diagnosed DM, we did not get a significant difference for any of the clinical parameters $(p>0.05)$. It indicates the association of CAM with hyperglycemia, rather than the duration of DM/status of 
Table 2 Comparison of patient's clinical data with respect to sugar levels $(\leq 200 \mathrm{mg} / \mathrm{dl} \mathrm{vs}>200 \mathrm{mg} / \mathrm{dl})$ and detection of DM (new vs old cases) in patients with CAM

\begin{tabular}{|c|c|c|c|}
\hline & & Blood sugar levels $(\leq 200 \mathrm{mg} / \mathrm{dl} \mathrm{vs}>200 \mathrm{mg} / \mathrm{dl})$ & $\mathrm{DM}($ Old vs Newly diagnosed $)$ \\
\hline Characteristics & No of patients (n) & $P$ value & $P$ value \\
\hline Primary symptoms & $\begin{array}{l}\text { Blindness (8) } \\
\text { Palatal ulcer (1) } \\
\text { Proptosis (4) } \\
\text { Loosening of teeth (5) } \\
\text { Orbital pain (1) } \\
\text { Headache (3) } \\
\text { Facial pain (11) } \\
\text { Facial swelling (10) } \\
\text { Facial numbness (3) }\end{array}$ & 0.811 & 0.096 \\
\hline Types of mucormycosis & $\begin{array}{l}\text { Rhino-orbital (33) } \\
\text { Rhino-cerebral (10) } \\
\text { Mandibular (1) } \\
\text { Sinonasal (2) }\end{array}$ & 0.849 & 0.869 \\
\hline Orbital extenteration & $\begin{array}{l}\text { Yes (8) } \\
\text { No (38) }\end{array}$ & 0.713 & 1.00 \\
\hline Surgical approach & $\begin{array}{l}\text { External (2) } \\
\text { Endoscopic (3) } \\
\text { Combined (37) } \\
\text { Medical treatment (2) }\end{array}$ & 0.520 & 0.568 \\
\hline Death of patients & During hospital stay (9) & 0.550 & 1.00 \\
\hline Hospital stay & During treatment (46) & 0.004 & 0.667 \\
\hline Revision surgery & During treatment $(31)$ & 0.673 & 0.697 \\
\hline
\end{tabular}

DM Diabetes Mellitus, CAM COVID Associated Mucormycosis

blood sugar that determines the clinical profile of the patients and treatment outcomes. We did not find any significant association between hyperglycemia and rhinocerebral mucormycosis with respect to the various subsites in the brain $(p>0.05)$. Again the duration of the disease did not have a direct association with the involvement of the orbital/brain. When the number of patients who underwent revision surgery was compared between the blood sugar levels/onset of DM, no significant difference was noted ( $p=0.673$ and 0.697 , respectively). The raised blood sugar during the second wave of COVID 19 infection could be due to the universal use of systemic steroids (dexamethasone/methylprednisolone) during the treatment of complicated COVID pneumonia. Although the systemic steroid is considered life-saving for the treatment of moderate to severe COVID pneumonia and has been shown to reduce mortality, it can potentiate a hyperglycemia state in such patients, predisposing them to life-threatening fungal infection. Again, there is an increased burden of DM in India in the past decade, where its prevalence is the secondhighest, affecting $8.9 \%$ of the adults with 77 million cases [17], significantly contributing to the incidence of CAM.
Different international and national guidelines have been proposed, including the dosing and duration of systemic steroids in the past 12 months for the better management of COVID pneumonia. According to the current guideline in India, patients with moderate pneumonia are advised for Intravenous methylprednisolone $0.5-1 \mathrm{mg} / \mathrm{kg} /$ day or dexamethasone $0.1-0.2 \mathrm{mg} / \mathrm{kg}$ for 3 days and for severe disease, methylprednisolone $1-2 \mathrm{mg} / \mathrm{kg} /$ day or dexamethasone $0.2-0.4 \mathrm{mg} / \mathrm{kg}$ is advised for 5-7 days. As recommended by the National Institute of Health(NIA), injection dexamethasone $(6 \mathrm{mg} / \mathrm{kg})$ is advised for minimum 10 days to the patients who require supplemented oxygen therapy or ventilator support [18]. Again, these treatment protocols are often customized in different tertiary care hospitals in India, where the dosing and duration of systemic steroids differ from one another. This difference in the protocol could be due to the variation in the infection rate and the availability of the basic infrastructure of the hospitals. According to the protocols, patients with mild COVID are always advised for medical treatment with self-isolation in the home, especially during the second wave of COVID 19. The systemic steroid is advised in 
moderate to severe diseases, which should only be started after the hospitalization of the patients where strict monitoring of the blood sugar and different inflammatory mediators can be undertaken. During the second wave of COVID 19 infection, there was a sudden increase in new cases, which was really a challenge to manage them in hospitals, especially in India with limited infrastructure, enforcing the patients for home isolation and self-administration of drugs. Often patients with mild COVID disease also takes systemic steroid because of anxiety and fear of the disease without even monitoring the blood sugar and oxygen saturation. Hence it could be a principal reason for the increase of cases of CAM during the second wave of COVID 19, where patients acquire the infection after the completion of the systemic steroid. Similar results had obtained in the present study where all the patients had a positive history of COVID 19 infection, although $41(89.13 \%)$ patients had a history of intake of systemic steroids. Hence the predisposing factors of CAM are still a debate in spite of adequate knowledge in current literature. The fungal infection itself causes a significant reduction in the lymphocyte count, especially the CD4 + and CD8 + T-cells, which may alter the innate immunity of the body predispose for the secondary fungal infection [19]. Again, the pathogenesis of the COVID 19 infection very much resembles the mucormycosis causing microangiopathy, angioinvasion, and endothelial damage aggravating the disease [20]. Finally, the universal use of glucocorticoids for the treatment of moderate to severe COVID infection is often responsible for invasive mucormycosis [21, 22]. In the present study, although majorities of articles have opined, uncontrolled diabetes mellitus being the major culprit for the CAM, 2 patients still did not have a history of DM as supported by the literature [6, 10]. Again, $5(10.86 \%)$ patients did not have a history of use of systemic steroids in spite of a documented history of COVID 19 infection. Hence the etiopathology of the pathology of mucormycosis is complex and later could be the combined effects of the dysregulated immune cell populations and the host response plays a critical role in the severity of the disease [23]. It is assumed that the COVID -19 infection itself could be a predisposing factor for the DM affecting the pancreases along with the systemic steroid, especially in middle-aged and non-diabetic patients. Although majorities of the articles have mentioned the association of blood sugar to mucormycosis, very few of them [4, 12] described the type, dose and duration of steroid used before the commencement of the fungal disease. Again, the exact correlation of the blood sugar level with the clinical profile and treatment outcomes in patients with CAM is ill-defined in the literature. As demonstrated in the present study, DM can be considered as the single most independent factor for the development of the CAM. Although blood sugar level does not significantly affect the clinical profile and treatment outcomes of the patients, a decreased preoperative blood sugar significantly decreases the hospital stay and hence postoperative morbidity. A large sample size may be required with a long-term follow-up period, maybe for a better understanding of various predisposing factors for the development of CAM.

\section{Conclusion}

The predisposing factors of invasive fungal disease in patients with COVID 19 infection is still a topic of debate because of the limited human understanding of the virus with the current literature. Though the use of systemic glucocorticoids is thought to be promising for the optimized treatment of moderate to severe COVID pneumonia, it is proved to be a double-aged sword potentiating a hyperglycaemic state in patients with COVID 19 infectioncausing CAM.

There were no significant differences in the clinical/ treatment outcomes in patients with CAM with respect to the level of initial blood sugar except for the hospital stay. Although the pathology of the disease has been explained in the past literature, affection of non-diabetic individuals/ absence of steroid use always questions the possibility of multifactorial association of dysregulated immune cell populations and the host response for CAM. A universal treatment guideline should be proposed, especially for the systemic steroid, to prevent its indiscriminate use in COVID infection. The basic infrastructure of the hospitals, especially the oxygenated hospital beds and the intensive care units, should be increased to accommodate all the moderate COVID infections, especially in the Indian subcontinent, to rationalize the medical treatment and decrease the secondary fungal infection associated with COVID 19. A large sample size may be required with a long-term follow-up period, maybe for a better understanding of various predisposing factors for the development of CAM.

Acknowledgements The authors would like to acknowledge that the study was conducted in All India Institute of Medical Sciences, Bhubaneswar, in the Department of ENT and Head Neck Surgery. There are no conflicts of interest among the authors. This Research has not been supported by any financial agency or funding organization.

Funding The study was not funded by any organization or institution.

\section{Declarations}

Conflicts of interest Disclosure of potential conflicts of interest: There are no conflicts of interest among the authors. 
Human or Animal Rights All procedures performed in studies involving human participants were in accordance with the ethical standards of the institutional and/or national research committee and with the 1964 Helsinki declaration and its later amendments or comparable ethical standards. Research involving human participants

Informed Consent Written informed consent has been taken from each patient prior to the surgery and same has been informed to the institute reviewer board. No part of the body has been demonstrated in the case report without the permission of the patient.

\section{References}

1. DeShazo RD (1998) Fungal sinusitis. Am J Med Sci 316:39-44

2. Gillespie MB, O'Malley BW (2000) An algorithmic approach to the diagnosis and management of invasive fungal rhinosinusitis in the immunocompromised patient. Otolaryngol Clin North Am 33:323-334

3. Jeong W, Keighley C, Wolfe R et al (2019) The epidemiology and clinical manifestations of mucormycosis: a systematic review and meta-analysis of case reports. Clin Microbiol Infect 25:26-34

4. Mehta S, Pandey A (2020) Rhino-Orbital Mucormycosis Associated With COVID-19. Cureus 12(9):e10726. https://doi.org/ 10.7759/cureus.10726.PMID:33145132;PMCID:PMC7599039

5. Mekonnen ZK, Ashraf DC, Jankowski T, Grob SR, Vagefi MR, Kersten RC, Simko JP, Winn BJ (2021) acute invasive rhinoorbital mucormycosis in a patient with COVID-19-associated acute respiratory distress syndrome. Ophthalmic Plast Reconstr Surg 37(2):40-80. https://doi.org/10.1097/IOP.00000000000 01889

6. John TM, Jacob CN, Kontoyiannis DP (2021) When uncontrolled diabetes mellitus and severe COVID-19 converge: the perfect storm for mucormycosis. J Fungi (Basel) 7(4):298. https://doi.org/10.3390/jof7040298.PMID:33920755;PMCID:PM C8071133

7. Sharma S, Grover M, Bhargava S, Samdani S, Kataria T (2021) Post coronavirus disease mucormycosis: a deadly addition to the pandemic spectrum. J Laryngol Otol 8:1-6. https://doi.org/10.10 17/S0022215121000992

8. Waizel-Haiat S, Guerrero-Paz JA, Sanchez-Hurtado L, CallejaAlarcon S, Romero-Gutierrez L (2021) A case of fatal rhinoorbital mucormycosis associated with new onset diabetic ketoacidosis and COVID-19. Cureus 13(2):e13163. https://doi. org/10.7759/cureus.13163.PMID:33575155;PMCID:PMC78701 13

9. Saldanha M, Reddy R, Vincent MJ (2021) Title of the article: paranasal mucormycosis in COVID-19 Patient. Indian J Otolaryngol Head Neck Surg 22:1-4. https://doi.org/10.1007/s1207 0-021-02574-0

10. Moorthy A, Gaikwad R, Krishna S, Hegde R, Tripathi KK, Kale PG, Rao PS, Haldipur D, Bonanthaya K SARS-CoV-2, uncontrolled diabetes and corticosteroids-an unholy trinity in invasive fungal infections of the maxillofacial region? A retrospective, multi-centric analysis

11. Karimi-Galougahi M, Arastou S, Haseli S (2021) Fulminant mucormycosis complicating coronavirus disease 2019 (COVID19). Int Forum Allergy Rhinol 11(6):1029-1030. https://doi.org/ 10.1002/alr.22785 (Epub 2021 Mar 13 PMID: 33713565)

12. Werthman-Ehrenreich A (2020) Mucormycosis with orbital compartment syndrome in a patient with COVID-19. Am J Emerg Med 42:264.e5-264.e8. https://doi.org/10.1016/j.ajem.20 20.09.032

13. Alekseyev K, Didenko L, Chaudhry B (2021) Rhinocerebral Mucormycosis and COVID-19 Pneumonia. J Med Cases 12(3):85-89. https://doi.org/10.14740/jmc3637

14. Revannavar SM, Supriya PS, Samaga L, Vineeth KV (2021) COVID-19 triggering mucormycosis in a susceptible patient: a new phenomenon in the developing world? BMJ Case Rep 14(4):e241663. https://doi.org/10.1136/bcr-2021-241663

15. Veisi A, Bagheri A, Eshaghi M, Rikhtehgar MH, RezaeiKanavi M, Farjad R (2021) Rhino-orbital mucormycosis during steroid therapy in COVID-19 patients: a case report. Eur J Ophthalmol 10:11206721211009450. https://doi.org/10.1177/11206721211 009450

16. Sen M, Lahane S, Lahane TP, Parekh R, Honavar SG (2021) Mucor in a viral land: a tale of two pathogens. Indian J Ophthalmol 69(2):244-252. https://doi.org/10.4103/ijo.IJO_3774_2 0.PMID:33463566;PMCID:PMC7933891

17. International Diabetes Federation (2020) https://idf.org/ournet work/ regions members/south-east-asia/members/94-india. html

18. Shah K, Dave V, Bradoo R, Shinde C, Prathibha M (2019) Orbital exenteration in rhino-orbito-cerebral mucormycosis: a prospective analytical study with scoring system. Indian J Otolaryngol Head Neck Surg 71:259-265

19. Gangneux JP, Bougnoux ME, Dannaoui E, Cornet M, Zahar JR (2020) Invasive fungal diseases during COVID-19: we should be prepared. J Mycol Med 30:100971

20. Sweeny JM, Barouqa M, Krause GJ, Gonzalez-Lugo JD, Rahman S, Gil MR.Evidence for secondary thrombotic microangiopathy in COVID-19. medRxiv preprint https://doi.org/10.1101/20 20.10.20.20215608

21. Clinical management protocol for COVID-19 (2020) https://www.mohfw.gov.in/pdf/Clin Accessed from 7 July 2020

22. The Recovery Collaborative Group (2020) Dexamethasone in Hospitalized Patients with Covid-19-Preliminary Report. NEngl J Med at NEJM@@@

23. Pasero D, Sanna S, Liperi C, et al. (2020) A challenging complication following SARS-CoV-infection: a case of pulmonary mucormycosis. Infection 1-6

Publisher's Note Springer Nature remains neutral with regard to jurisdictional claims in published maps and institutional affiliations. 